\title{
Scheduling of Periodic Packets in Energy-Aware Wireless Networks
}

\author{
Xiao Qin*, Mohammed Alghamdi, Mais Nijim, Ziliang Zong, and Kiranmai Bellam \\ Department of Computer Science \\ New Mexico Institute of Mining and Technology \\ 801 Leroy Place, Socorro, New Mexico 87801-4796 \\ (xqin, alghamdi, mais, zzong, kiranmai)@cs.nmt.edu
}

\begin{abstract}
Existing packets scheduling algorithms designed for energy-efficient wireless networks ignore important features of periodic packets, thereby being inadequate for periodic packets with energy constraints. To remedy this problem, we present in this paper an approach to scheduling periodic packets in wireless networks subject to both timing and energy constraints. We propose a necessary and sufficient feasibility check for $a$ set of periodic packets to be transmitted over a wireless link. Next, we develop an algorithm to schedule periodic packets (or ESPP for short) over a wireless link. The ESPP algorithm aims at minimizing energy dissipation of periodic packets without missing deadlines of periodic packets. We show through simulation studies that ESPP can significantly reduce energy consumption of wireless networks by an average of $46.4 \%$ while guaranteeing timing constraints of periodic packets.
\end{abstract}

\section{INTRODUCTION}

Wireless networks have been widely applied to support real-time applications like interactive multimedia and video-on demand, which depend not only on the logical results of computation, but also on the time at which the results are produced [2][9][14]. A primary concern in real-time applications in wireless networks is energy efficiency, since a majority of wireless devices operate on batteries that need to be regularly recharged from a power source [3][6][9][12]. The lifetimes of wireless networks largely depend on energy, because wireless devices may not be immediately charged once energy is drained. Thus, it is important to develop energy conservation techniques to achieve long battery life for wireless networks supporting real-time applications.

Some research has been directed at developing realtime packet scheduling algorithms [2][4][8]. Although previous work focused on improving performance for wireless networks, the motivation of the previous research is not to conserve energy in wireless networks.

There has been a lot of research on energy saving strategies for wireless networks [6]. Stine and Veciana studied various methods to improve energy efficiency of centrally con-trolled wireless data networks [11]. Wang et al. proposed a new adaptive call admission control scheme that takes energy conservation in wireless networks into account [13]. While this previous work can minimize energy consumption in wireless networks, it is not adequate for real-time wireless networks due to the lack of ability to deal with timing constraints.

Recently, many solutions were proposed to incorporate energy conservation schemes into a variety of real-time applications. Havinga and Smit identified prominent problems of wireless multimedia networking and pre-sented solutions to improve energy efficiency for multi-media applications [5]. UysalBiyikoglu et al. studied the issue of minimizing energy subject to a deadline or a delay constraint in wireless networks [12]. In our previous work, we proposed an energy-aware scheduling algorithm to generate schedules that minimize both energy consumption and the probability of missing deadlines for real-time

\footnotetext{
* Corresponding author. http://www.cs.nmt.edu/ xqin This paper appeared in the Proceedings of the 26th IEEE International Performance Computing and Communications Conference (IPCCC'07), New Orleans, Louisiana, April 2007.
} 
packets [3]. Although the energy-aware packet scheduling issue in the context of wireless networks was addressed, an issue of energy-efficient scheduling for periodic packets over wireless networks was not considered.

Minimizing energy required to transmit periodic packets over a wireless network is of critical importance, because the wireless network has to handle packets from both periodic and non-periodic sources [7][15]. In this paper, we consider the problem of minimizing energy consumed by a node on a point-topoint wireless link used to transmit periodic packets. Our algorithm aims to minimize transmission energy of a set of periodic packets without missing deadlines. To achieve this goal, the propose scheme reduces power levels for periodic packets in a judicious way that the lowered power levels can efficiently lead to energy saving in the wireless link. We simulate a wireless link and compare our approach with a baseline algorithm that ignores the issue of energy efficiency as a primary design goal.

The rest of the paper is organized in the following way. Section II describes the problem statement and system model. Section III proposes a scheduling algorithm for periodic packets over a wireless link. Performance evaluation of the proposed algorithm is presented in Section IV. Finally, Section V concludes the paper with summary and future research directions.

\section{MAThematical Models}

Since a wide variety of real-time communicationintensive applications are periodic in nature, we consider in this study the issue of energy-aware scheduling for periodic packets in wireless networks. Without loss of generality, we are focused on packet scheduling between two nodes in a wireless network. However, the proposed scheme can be readily applied to any wireless network containing a large number of nodes in a complex environment.

A periodic packet in our model is characterized as a set of two parameters, i.e., $P_{i}=\left(s_{i}, p_{i}\right)$, where $s_{i}$ is the data size measured in $\mathrm{KB}$, and $p_{i}$ is the period. It is worth noting that the two parameters of each periodic packet obtained from an application are specified by users. To simplify the presentation of our model, we assume that the first instance of packet $P_{i}$ arrives at time 0 . A new packet instance is generated every $p_{i}$ time units, and the $j$ th instance of $P_{i}$ will be arriving at time $(j-1) p_{i}$. The deadline of the $j$ th packet instance equals to the arrival time of the next packet instance, i.e., the $j$ th must be delivered before time $j p_{i}$. A periodic packet is said to be feasible if all its packet instances can be transmitted before their corresponding deadlines. Given a set of $n$ periodic packets, i.e., $P=\left\{P_{1}, P_{2}, \ldots P_{n}\right\}$, there exists a feasible schedule for the packet set if and only if there is a feasible schedule for the planning cycle of the packets. Note that the planning cycle $p$ is the least common multiple of all the packets' periods.

Let $t_{i j}$ denote the trans-mission time of the $j$ th instance of packet $P_{i}$, then $t_{i j}$ can be expressed as

$$
t_{i j}=s_{i} / b_{i j},
$$

where $b_{i j}$ is the transmission rate of the $j$ th packet instance of $P_{i}$. To guarantee that a periodic packet $P_{i}$ is feasible, we have to ensure that each instance of the packet is transmitted in a timely manner. Thus, we have

$$
\forall j: s_{i} / b_{i j} \leq p_{i}
$$

It is supposed that the transmission rate can be dynamically changed between a minimum rate $b_{\min }$ and a maximum rate $b_{\max }$. With respect to energy conservation in wireless networks, a transmission rate corresponds to a energy consumption rate measured by Joule/bit. Thus, $b_{\min }$ corresponds to the minimum energy consumption rate $\theta_{\min }$, whereas $b_{\max }$ corresponds to the maximum energy consumption rate $\theta_{\max }$. The energy consumption rate $\theta_{i j}$ of the $j$ th instance of packet $P_{i}$ is a function of the transmission rate $b_{i j}$. $\theta_{i j}$ can be written as [3]

$$
\theta_{i j}=\Gamma\left(b_{i j}\right)
$$

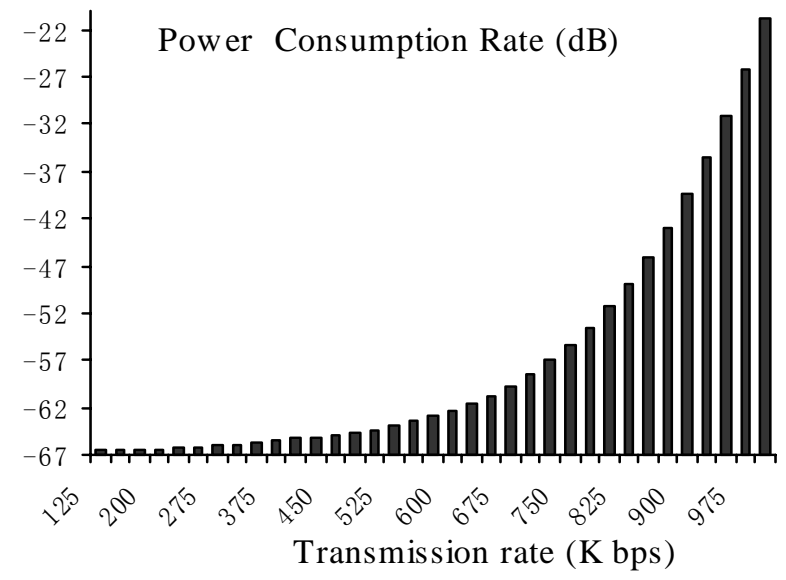

Fig. 1. Energy consumption model 
Fig. 1 depicts the relationship between the power consumption rate and the message transmission rate. The vertical axis is the power consumption rate, whereas the horizontal axis is the message transmission rate measured in Kbps. We built the energy consumption model based on the empirical results reported in [13]. We extended the results presented in [13] using curve fitting so that we can quantitatively obtain corresponding power consumption when message transmission rate varies in the range between $125 \mathrm{kbps}$ and $1000 \mathrm{kbps}$.

Now we consider energy consumption incurred by a set $P=\left\{P_{1}, P_{2}, \ldots P_{n}\right\}$ of $n$ periodic packets. To efficiently reduce energy consumption of the periodic packet set while guaranteeing timing constraints, we leverage the following function to quantitatively measure energy consumption caused by a packet instance. The energy consumption $\psi_{i j}$ of the $j$ th instance of packet $P_{i}$ is calculated as a product of the data size of the instance and the energy consumption rate. Hence, we have the following equation where $\theta_{i j}$ can be derived from Eq. (2)

$$
\psi_{i j}=S_{i \times} \theta_{i j}
$$

Given a periodic packet $P_{i}$, the energy consumption of the periodic packets during the planning cycle can be written as

$$
\begin{aligned}
& \psi_{i}=\sum_{j=1}^{p / p_{i}}\left(s_{i} \cdot \theta_{i j}\right), \\
& \text { subject to } \forall 1 \leq j \leq \frac{p}{p_{i}}: \frac{s_{i}}{\Gamma^{-1}\left(\theta_{i j}\right)} \leq p_{i}, \\
& \forall 1 \leq j \leq \frac{p}{p_{i}}: \theta_{\min } \leq \theta_{i j} \leq \theta_{\max } .
\end{aligned}
$$

where $p$ is the least common multiple of all the packets' periods, $\frac{s_{i}}{\Gamma^{-1}\left(\theta_{i j}\right)} \leq p_{i}$ is the transmission time of the $j$ th instance.

We can calculate the energy consumption of the set of $n$ periodic packets in the planning cycle $p$ using Eq. (8) as below

$$
\begin{aligned}
\psi & =\sum_{i=1}^{n} \psi_{i} \\
& =\sum_{i=1}^{n} \sum_{j=1}^{p / p_{i}}\left(s_{i} \cdot \theta_{i j}\right) .
\end{aligned}
$$

Now we formulate the energy conservation problem as follow,

$$
\text { Minimize } \psi=\sum_{i=1}^{n} \sum_{j=1}^{p / p_{i}}\left(s_{i} \cdot \theta_{i j}\right)
$$

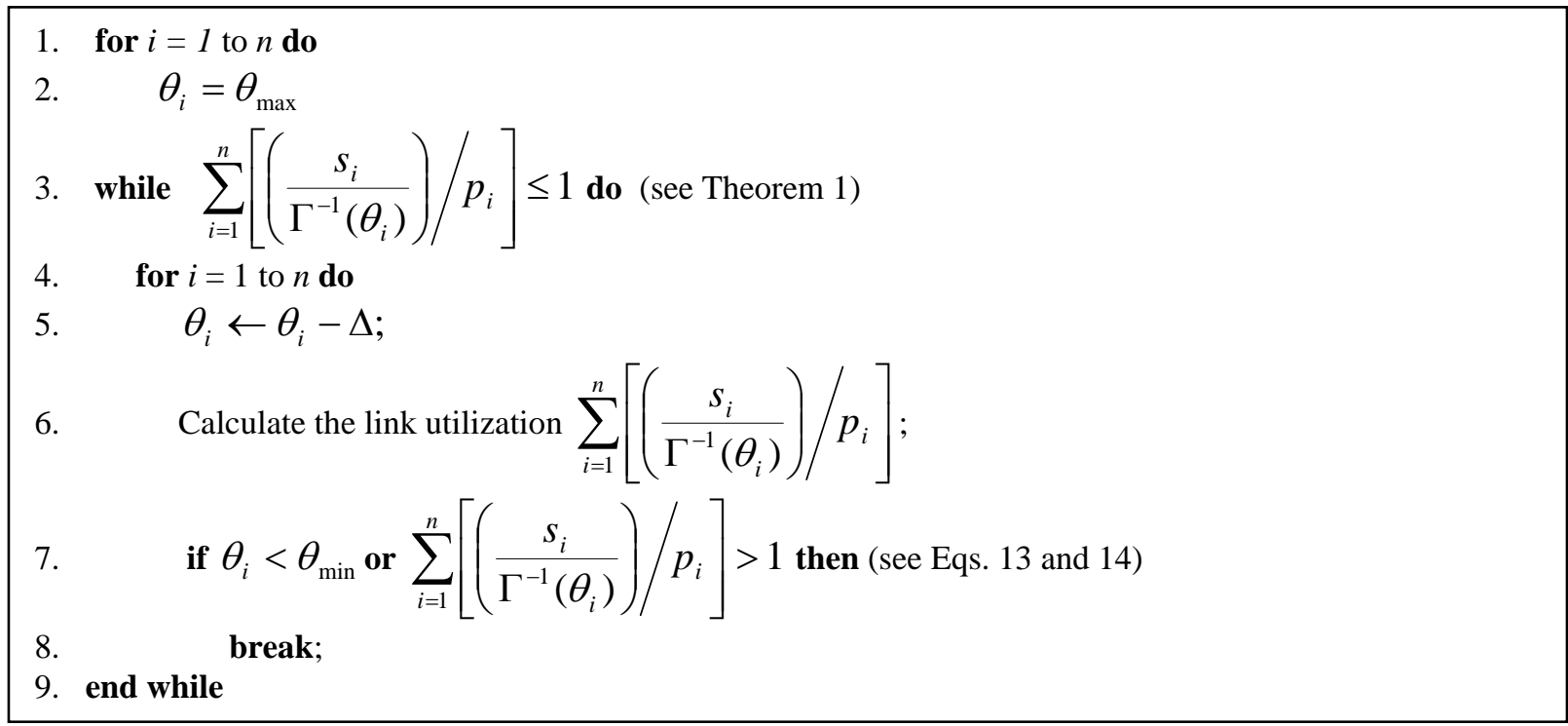

Fig. 2. The ESPP scheduling algorithm for periodic packets transmitted over a point-to-point wireless link 


$$
\begin{gathered}
\text { Subject to } \forall 1 \leq j \leq \frac{p}{p_{i}}: \frac{s_{i}}{\Gamma^{-1}\left(\theta_{i j}\right)} \leq p_{i} \\
\forall 1 \leq j \leq \frac{p}{p_{i}}: \theta_{\min } \leq \theta_{i j} \leq \theta_{\max } .
\end{gathered}
$$

where the timing constraint encodes the fact that all packets must be transmitted before their deadlines.

\section{THE ESPP ALgORITHM}

To reduce energy consumption of periodic packets in a planning cycle, we propose in this section an energy-aware scheduling algorithm, or ESPP for short. The ESPP algorithm (the pseudo code is shown in Fig. 3) aims at minimizing energy dissipation experienced by a wireless network link (see Eq. 7).

In an effort to minimize energy consumption caused by periodic packets, the ESPP algorithm manages to decrease transmission rates for periodic packets within a planning cycle in a judicious way that packets can be delivered before their deadlines. The decreased transmission rates in turn result in reduced energy dissipation.

To efficiently determine optimal transmission rate, we develop an approach to checking feasibility of a set of periodic packets. The approach can be applied to verify that if all the periodic packets can be sent via a wireless link at minimized transmission rates while having their timing constraints guaranteed. A necessary and sufficient feasibility check is presented in Theorem 1 as follows.

Theorem 1. Given a set $P=\left\{P_{1}, P_{2}, \ldots P_{n}\right\}$ of $n$ periodic packets, all packets can be feasibly scheduled on a wireless link if and only if the total utilization is equal to or less than one. That is,

$$
\sum_{i=1}^{n}\left[\left(\frac{s_{i}}{\Gamma^{-1}\left(\theta_{i}\right)}\right) / p_{i}\right] \leq 1,
$$

Proof: It has been proved in the necessary and sufficient feasibility check for a packet set under ideal EDF scheduling requires the total of the worst-case utilizations be equal to or less than one, e.g., $\sum_{i=1}^{n} t_{i} / p_{i} \leq 1$, where $t_{i}$ is the transmission time of a packet. $t_{i}$ equals to $\frac{s_{i}}{\Gamma^{-1}\left(\theta_{i}\right)}$, where $\theta_{i}$ is the energy consumption rate for packet $P_{i}$. After substituting $\frac{s_{i}}{\Gamma^{-1}\left(\theta_{i}\right)}$ for $t_{i}$, we obtain the necessary feasibility test for security sensitive tasks as

$\sum_{i=1}^{n}\left[\left(\frac{s_{i}}{\Gamma^{-1}\left(\theta_{i}\right)}\right) / p_{i}\right] \leq 1$

Theorem 1 clearly suggests that the total processor utilization can not exceed the available wireless link capacity if there exists at least one feasible schedule for the set of periodic packets. Based on the previous theorem, it is clear that Corollary 1 holds.

Corollary 1. Given a set $P=\left\{P_{1}, P_{2}, \ldots P_{n}\right\}$ of $n$ periodic packets, if $\sum_{i=1}^{n}\left[\left(\frac{s_{i}}{\Gamma^{-1}\left(\theta_{\max }\right)}\right) / p_{i}\right]>1$, there is no feasible schedule for $P$.

Without loss of generality, we assume the energy consumption rates of all instances of a periodic are identical, i.e., $\theta_{i}=\theta_{i 1}=\theta_{i 2}=\ldots=\theta_{i r}, r=p / p_{i}$. This assumption is reasonable, because Theorem 2 indicates that if a feasible schedule exist for a set of periodic packet, then we can be generated a feasible schedule under a set of energy consumption rates $\theta=\left\{\left(\theta_{1 j}\right), \ldots,\left(\theta_{n j}\right)\right\}$, where

$\theta_{i 1}=\ldots=\theta_{i r}, r=p / p_{i}$.

Theorem 2. Given a set $P=\left\{P_{1}, P_{2}, \ldots P_{n}\right\}$ of $n$ periodic packets, and a set of energy consumption rates $\theta^{\prime}=\left\{\left(\theta_{1 j}^{\prime}\right), \ldots,\left(\theta_{n j}^{\prime}\right)\right\}$, if there exist a feasible schedule under $\theta^{\prime}$ that minimize energy dissipation, then we also can generate a feasible schedule under $\theta=\left\{\left(\theta_{1 j}\right), \ldots,\left(\theta_{n j}\right)\right\}$ and $\theta_{i}=\theta_{i 1}=\ldots=\theta_{i r}=\sum_{j=1}^{r} \theta_{i j}^{\prime} / r \quad\left(r=p / p_{i}\right)$ that can also minimize energy consumption.

Proof: Since $\theta_{i}$ is the mean of $\theta_{i 1}, \ldots, \theta_{i r}$, $\theta=\left\{\left(\theta_{1 j}\right), \ldots,\left(\theta_{n j}\right)\right\}$ satisfies the constraint of energy consumption rates (see Eqs. 7 and 11). Because we 
have $\sum_{j=1}^{r} \frac{s_{i}}{\Gamma^{-1}\left(\theta_{i j}\right)}=\sum_{j=1}^{r} \frac{s_{i}}{\Gamma^{-1}\left(\theta^{\prime}{ }_{i j}\right)}=r \frac{s_{i}}{\Gamma^{-1}\left(\theta_{i}\right)}$, it

shows that replacing $\theta$ with $\theta$ ' has no impact on the capacity constraint. Thus, a feasible schedule under $\theta=\left\{\left(\theta_{1 j}\right), \ldots,\left(\theta_{n j}\right)\right\}$ can also minimize energy dissipation.

Based on Theorem 2, we can easily prove that given a a set $P=\left\{P_{1}, P_{2}, \ldots P_{n}\right\}$ of $n$ periodic packets, there exists an optimal feasible schedule where the energy consumption rate of the ith packets is a constant at every instance.

Theorem 3. Given a set $P=\left\{P_{1}, P_{2}, \ldots P_{n}\right\}$ of $n$ periodic packets, there exists a feasible schedule where (1) the energy consumption is minimized, and (2) the energy consumption rate of $P_{i}$ is constant at every instance.

Proof: First, the capacity constraint is satisfied, e.g., $\sum_{i=1}^{n}\left[\left(\frac{s_{i}}{\Gamma^{-1}\left(\theta_{i}\right)}\right) / p_{i}\right] \leq 1$, which means that an EDF scheduling [3] can be used to generate a feasible schedule for the set of packets. Thus, the timing constraints are satisfied (see Eq. 9). Further, Theorem 2 proves that we can generate an optimal schedule where the energy consumption rate of $P_{i}$ is constant at every instance. Therefore, we prove that there is an optimal feasible schedule where the energy consumption rate of the $P_{i}$ is a constant at every instance.

In light of Theorem 3, we have Corollary2, which indicates that choosing appropriate energy consumption rate for each periodic packet is a way of minimizing energy dissipation of the packet set.

Corollary 2. Given a set $P=\left\{P_{1}, P_{2}, \ldots P_{n}\right\}$ of $n$ periodic packets, and a set of energy consumption rates $\theta=\left\{\left(\theta_{1 j}\right), \ldots,\left(\theta_{n j}\right)\right\}$, the objective function of the proposed ESPP algorithm is:

$$
\begin{aligned}
& \text { Minimize } \psi=\sum_{i=1}^{n} \frac{p}{p_{i}}\left(s_{i} \cdot \theta_{i}\right), \\
& \text { Subject to } \forall 1 \leq j \leq \frac{p}{p_{i}}: \theta_{\min } \leq \theta_{i} \leq \theta_{\text {max }}, \\
& \qquad \sum_{i=1}^{n}\left[\left(\frac{s_{i}}{\Gamma^{-1}\left(\theta_{i}\right)}\right) / p_{i}\right] \leq 1 .
\end{aligned}
$$

Now we are positioned to delineate the ESPP scheduling algorithm for periodic packets transmitted over a wireless link. To guarantee timing constraints, the ESPP algorithm first makes an attempt to send all the packets at the highest transmission rates, meaning that the energy consumption rate of each periodic packet is set to the maximum value. In other words, ESPP initially endeavors to achieve a high schedulability. The goal of Steps 3-8 is to gradually decrease energy consumption rate by lowering power level provided that the total link utilization is less than or equal to 1 (see Theorem 1). As such, transmission energy is conserved by reducing energy consumption rate and transmitting periodic packets over a longer amount of time. Step 6 is performed to quantitatively calculate the link utilization. In doing so, the ESPP algorithm is able to substantially reduce transmission energy consumed by the periodic packets while guaranteeing that all the periodic packets can be delivered before their deadlines. Since there are $n$ periodic packets in the set, Steps 4 and 5 repeatedly reduces the energy consumption rate for all the periodic packets. The ESPP algorithm stops when Step 7 detects that the utilization is greater than 1 . The time complexity of the ESPP algorithm is analyzed as follows.

Theorem 4. The time complexity of ESPP is $\mathrm{O}\left(n^{2} m\right)$ where $n$ is the number of periodic packets and $m$ is the number of times Step 3 is repeated.

Proof: Step 1 takes time $\mathrm{O}(n)$ to initialize power levels of all periodic packets. The time complexity of Step 6 to calculate a link utilization is $O(n)$. Steps $4-8$ take time $\mathrm{O}\left(n^{2}\right)$ to gradually reduce power levels of the periodic packets. Since Step 3 is repeatedly executed $\mathrm{m}$ times, the time complexity of the ESPP algorithm is $\mathrm{O}\left(n^{2} m\right)$.

\section{Simulation STUdies}

The performance of the ESPP algorithm is quantitatively evaluated using simulations. In this study we compared ESPP with a baseline algorithm: MAX-EDF. The alternative ignores the issue of energy efficiency as a primary design goal, because it does not vary power levels and transmission times for periodic packets. Thus, MAX-EDF transmits periodic packets using the highest transmission rate. Note that the highest transmission rate in this study is set to 1000 kbps. The MAX-EDF algorithm picks a ready packet with the earliest deadline to transmit. 
Table I. Simulation Parameters

\begin{tabular}{|l|l|}
\hline \multicolumn{1}{|c|}{ Parameter } & \multicolumn{1}{|c|}{ Values } \\
\hline Networks bandwidth & $125-1000 \mathrm{Kbps}$ \\
Minimal period & $5 \mathrm{Sec}$. \\
Maximal period & $10,20, \ldots, 60 \mathrm{Sec}$. \\
Data size & $20-100 \mathrm{~KB}$ \\
Number of periodic packets & 10 \\
\hline
\end{tabular}

The simulation parameters used in our simulation studies are shown in Table I. The values of the simulation parameters are chosen in a way that the link utilization for MAX-EDF is always less than 1 . This is because if MAX-EDF is unable to generate a feasible schedule to guarantee deadlines of periodic packets, neither can ESPP produce any feasible schedule.

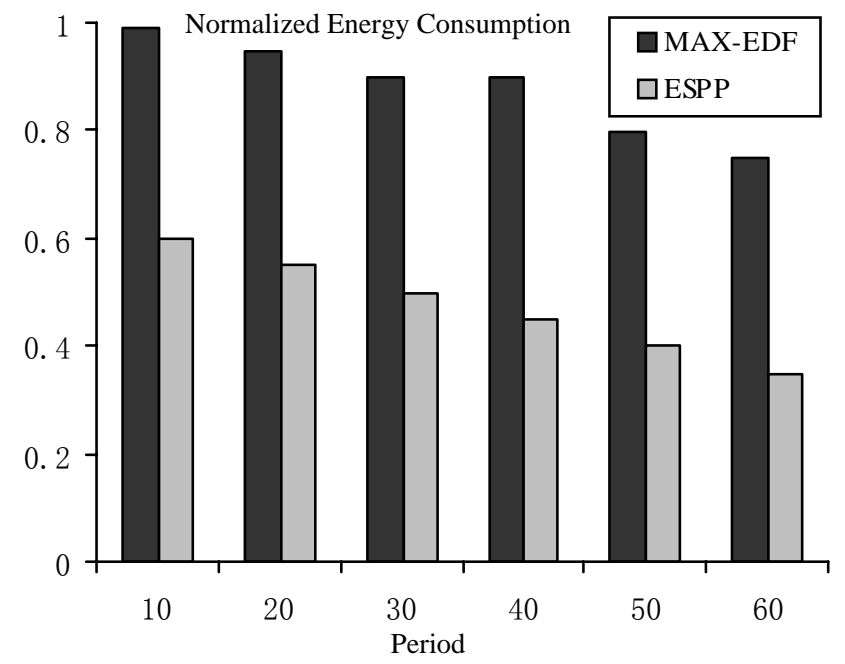

Fig. 3. Normalized energy consumption of a wireless link using ESPP and MAX-EDF algorithms to transmit periodic packets.

Fig. 3 shows normalized energy required by ESPP and MAX-EDF to transmit a set of periodic packets over a point-to-point wireless link. In this experimental, we focus on impacts of periods on energy consumption. Therefore, we vary the periods of packets from 10 to $60 \mathrm{Sec}$. with increments of $10 \mathrm{Sec}$, whereas we fix the values of data size and number of periodic packets.

First, Fig. 3 reveals that the ESPP algorithm significantly reduces energy consumption caused by transmitting a set of periodic packets over the simulated wireless link. Compared with MAX-EDF, the proposed ESPP reduces the transmission energy consumption by an average of $46.4 \%$. We attribute this energy conservation achieved by ESPP to the virtue of fact that ESPP decreases power levels for periodic packets in a judicious way while guaranteeing the deadlines of periodic packets by keeping the link utilization below 1 .

Second, Fig. 3 shows that the normalized energy consumption experienced by the wireless link decreases with the increasing value of period. This is mainly because when the period is increased, the number of packets to be transmitted within a given amount of time (i.e., planning cycle) drops, meaning that energy required to send the decreasing number of packets goes down accordingly.

Last but not least, Fig. 3 illustrates that transmission energy saving by our ESPP become more pronounced when the periods are increased. For example, ESPP conserves energy by $39.4 \%$ when the period is 10 Sec., whereas the reduction in energy consumption becomes $46.4 \%$ when the period is set to $60 \mathrm{Sec}$. We can explain this result by the fact that increasing periods gives rise to smaller link utilization, which in turn offers more opportunities for ESPP to further decrease power levels to save energy. This result suggests that ESPP can conserve more energy for periodic packets with large periods.

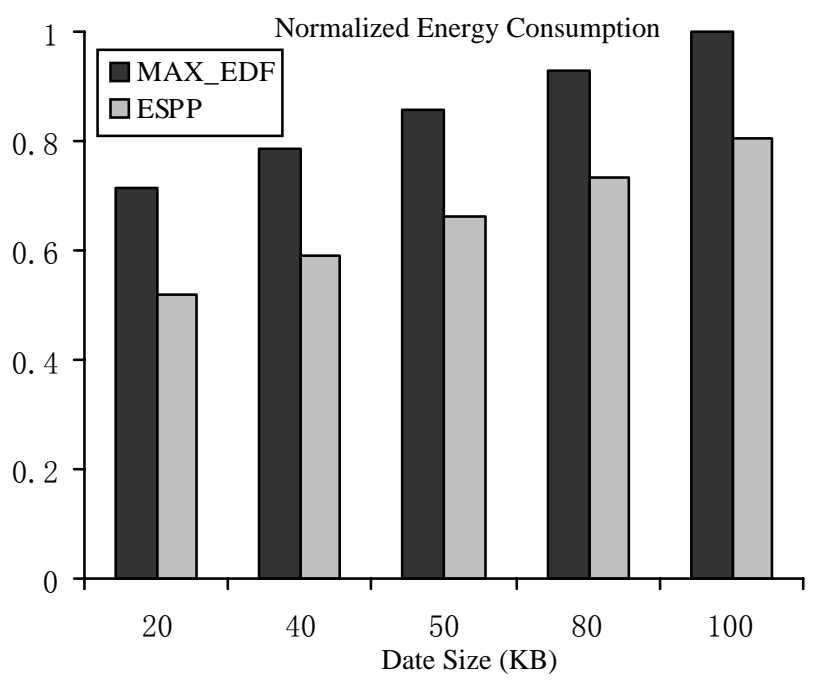

Fig. 4. Impact of data size on normalized energy consumption of a wireless link.

In the second experiment, we study the performance impact of data size on energy consumption. The data size is varied from 20 to $100 \mathrm{~KB}$, while other simulation parameters are fixed. First, Fig. 4 demonstratively shows that ESPP efficiently minimizes transmission energy, and this result is consistent with 
those reported in Fig. 3. Second, Fig. 4. shows the normalized energy consumption increases when the data size goes up. This result is expected because transmission energy heavily depends on the data size (see Eq. 8), meaning that larger the periodic packets cause more energy dissipation. More interestingly, the performance improvement in energy saving becomes less dramatic with the increasing value of the data size. For example, the reduction in energy consumption is $27.6 \%$ when data size is set to $20 \mathrm{~KB}$, and the reduction in transmission energy drops to $19.7 \%$ when the period is $100 \mathrm{~KB}$. The implication behind this finding is that smaller periodic packets can benefit more in terms of energy conservation from the proposed ESPP.

\section{SUMMARY AND FUTURE WORK}

A primary concern in packet transmission over wireless networks is energy efficiency, since a majority of wireless devices operate on batteries that need to be regularly recharged from a power source. Existing energy-efficient packet scheduling schemes found in the literature only consider non-periodic packets. In this paper, we have focused on a novel energy-efficient scheduling algorithm called ESPP for periodic packets over wireless networks. ESPP is conducive to minimizing transmission energy of a set of periodic packets over a point-to-point wireless link without missing deadlines of the packets. Energy is efficiently conserved by ESPP by reducing power levels and transmitting periodic packets over an increased amount of time. We simulated a wireless link and compared our approach with a baseline algorithm that ignores the issue of energy efficiency as a primary design goal. Experimental results show that the proposed ESPP scheme reduces energy consumption by an average of $46.4 \%$ without violating timing constraints of periodic packets.

Several intriguing problems remain open in the present context. The following two are of particular interests. First, the issue of how much energy can be conserved by ESPP in a large-scale wireless a network has not been addressed in this study. Second, the problem of how to integrate ESPP into a system-wide wireless network has not been studied in this paper. Apart from addressing these two issues, in the future work we intend to investigate energy-efficient solutions for embedded storage systems.

\section{ACKNOWLEDGEMENTS}

The work reported in this paper was supported in part by the New Mexico Institute of Mining and Technology under Grant 103295 and by Intel Corporation under Grant 2005-04-070.

\section{REFERENCES}

[1] T.F. Abdelzaher and K.G. Shin., "Combined Task and Message Scheduling in Distributed Real-Time Systems," IEEE Trans. Parallel and Distributed Systems, Vol. 10, No. 11, Nov. 1999.

[2] M Adamou, S Khanna, I Lee, I Shin, S Zhou, "Fair Real-time Traffic Scheduling over a Wireless LAN," Proc. IEEE Symp. Real-Time Systems, 2001.

[3] M. Alghamdi, T. Xie, and X. Qin, "PARM: A Power-Aware Message Scheduling Algorithm for Real-Time Wireless Networks," Proc. ACM Int'l Workshop Wireless Multimedia Networking and Performance Modeling, Oct. 2005.

[4] S. Choi and K. G. Shin, "A Unified Wireless LAN Architecture for Real-time and Non-Realtime Com-munication Services," ACM Trans. Networking, Feb. 2000.

[5] M. Havinga and G. Smit, "Energy-efficient wireless networking for multimedia Applications," Wireless communications and Mobile Computing, Wily, pp.165-184, 2001.

[6] C. Jones, “A Survey of Energy Efficient Network Protocols for Wireless Networks," Wireless Networks, pp.343-358, 2001.

[7] S. R. Li, "Considerations of Periodic Traffic in Packet Switching," IEEE Trans. Communications, Vol.38, No.9, pp. 1625-1629, Sept. 1990.

[8] S. Lu, V. Bharghavan, and R. Srikant, "Fair Scheduling in Wireless Packet Networks," IEEE/ACM Trans. Networking, vol.7, no.4, Aug. 1999.

[9] P. Nuggehalli, V. Srinivasan, and R. R. Rao, "Energy Efficient Transmission Scheduling for Delay Constrained Wireless Networks," IEEE Trans Wireless Communications, Vo.5, No.2, pp.531-539, 2006.

[10] J. A. Stankovic, M. Spuri, K. Ramamritham, G.C. buttazzo, "Deadline Scheduling for real-time system - EDF and related Algorithms," Kluer Academic Publishers, 1998.

[11] J. A. Stine, G.Veciana, "Improving Energy Efficiency of Centrally Controlled Wireless Data Networks,” Wireless Networks, 681-700, 2002.

[12] E. Uysal-Biyikoglu, B. Prabhakar, and A. E. Gamal, "Energy-Efficient Packet Transmission over a Wireless Link,” IEEE Trans. Networking, 
Vol.10, No.4, pp.487-499, 2002.

[13] X. Wang, and W. Wang, "Energy-Aware Call Admission Control Scheme in Wireless Cellular Networks," Proc. IEEE Globecom, 2004.

[14] T. Xie and X. Qin, "A New Allocation Scheme for Parallel Applications with Deadline and Security Constraints on Clusters,” Proc. IEEE Int'l Conf. Cluster Computing, Sept. 2005.

[15] T. Xie and X. Qin, "Improving Security for Periodic Tasks in Embedded Systems through Scheduling,” ACM Trans. Embedded Computing Systems, 2007. 